\title{
ÜBER DIE KEIMESENTWICKLUNG IN « TAUBEN » (ABORTIVEN) BIENENEIERN
}

\section{Sur le développement embryonnaire dans les œufs d'abeille abortifs}

\author{
von W. FYG
}

Bubenbergstrasse 27, 3700 Spiez (Schweiz)

\section{SUMMARY}

ON THE EMBRYONIC DEVELOPMENT IN ABORTIVE BEE'S EgGS

The anomalous embryonic development in sterile bee's eggs is described by means of original drawings. The investigations showed that, in a great number of abortive eggs, the first symptoms of misdevelopment are evident already during cleavage (fig. 2-5). Partial embryos of different sizes are frequently occurring (fig. 7-11), and not seldom almost complete, but incapable of hatching embryos appear.

Obviously the crisis of development in abortive eggs occurs at different times. Individually, the causes are still unknown; the anomalies, however, seem to be hereditary. Certainly, these symptoms are not caused by exterior factors.

\section{ZUSAMMENF ASSUNG}

An Hand von Originalzeichnungen wird die abnorme Embryonalentwicklung in " tauben 》 Bieneneiern beschrieben. Die Untersuchungen ergaben, daß die ersten Anzeichen einer Fehlentwicklung in vielen tauben Eiern bereits zur Zeit der Furchung festzustellen sind (Abb. 2-5). Oft entstehen unterschiedlich grosse Teilkeime (Abb. 7-11), nicht selten fast fertige, aber schlüpfunfähige Embryonen (Abb. 12).

Offenbar tritt die Entwicklungskrise in den tauben Bieneneiern zu verschiedener Zeit ein. Die Ursachen sind im einzelnen noch unbekannt, doch scheinen die Anomalien erblich bedingt zu sein. Mit Sicherheit handelt es sich nicht um eine durch äussere Faktoren verursachte Erscheinung. 


\section{I. - EINLEITUNG}

Es gibt junge, begattete Bienenköniginnen, die vorwiegend oder ausschliesslich Eier legen, aus welchen keine oder nur vereinzelt Larven schlüpfen. Diese Eier werden von den Imkern als “ taub ) und in der Fachliteratur als abortiv oder steril bezeichnet. Sie unterscheiden sich, frisch abgelegt, weder in der Form und Grösse, noch in der Farbe von normalen Bieneneiern. Nach einigen Tagen schrumpfen sie dann allerdings oft etwas ein, werden aber in der Regel von den brutpflegenden Bienen trotzdem in den Waben belassen.

Einzelne Bienenzüchter kannten diese Anomalie offenbar schon in der zweiten Hälfte des letzten Jahrhunderts. Si weurde erstmals kurz von KLEINE (1861) und dann ausführlicher von Herlikofer (1864) beschrieben. Später beschäftigten sich auch die Zoologie-Professoren v. Siebold (1871), Craus (1873) und Leuckart $(1871,1875)$ damit. Entgegen der damals vorherrschenden Meinung konnte Herlikofer mit ebenso einfachen wie zweckmässigen Versuchen zeigen, dass die mangelhafte Entwicklung der Eier nicht durch eine ungenügende Pflege des Geleges verschuldet wird, sondern irgendwie mit der Königin zusammenhängen muss. Er gab einem Bienenvolk mit einer solchen Bienenkönigin, deren Eier sich als entwicklungsunfähig erwiesen hatten, ein Wabenstück mit normalen Eiern zur Bebrütung und stellte fest, dass sie sich alle gut entwickelten. Umgekehrt erhielt ein anderes, starkes Bienenvolk, welches reichlich eigene gesunde Brut besass, einen Wabenausschnitt mit tauben Eiern zur Aufzucht; sie entwickelten sich in diesem Volk auch nicht. Diese Versuche, die von Hiтcнсоск (1956) mit dem gleichen Ergebnis mehrmals wiederholt wurden, beweisen hinreichend, dass die Ursache für die mangelnde Entwicklung der Eier nicht bei den Pflegebienen, sondern bei der Königin zu suchen ist.

Verschiedentlich wurde beobachtet, dass sich die unbefruchteten, haploiden Eier von derartigen Bienenköniginnen parthenogenetisch zu normalen Drohnen zu entwickeln vermögen (BETTs, 1923/24, 1926; FYg, 1936 und spätere, unveröffentlichte Befunde; Geiger, 1943). Man muss sich deshalb fragen, ob die Fehlentwicklung der tauben Bieneneier zur Eibefruchtung in ursächlicher Beziehung steht und auf letalen Genkombinationen oder zygotischen Letalfaktoren (HADoRN, 1955) beruht. Obwohl in neuerer Zeit auch bei der Honigbiene verschiedene letale Erbfaktoren nachgewiesen worden sind ${ }^{1}$, lässt sich diese Frage heute noch nicht beantworten (RuTtwer, 1968).

Auf ganz andere Weise hat Flanders (1959) die Anomalie zu erklären versucht. Er glaubt, dass die mangelnde Entwicklungsfähigkeit der tauben 1962.

1. Mackensen, 1951, 1955 ; Laidlaw, Gomes et Kerr, 1956 ; Hachinohe et Jimbu, 1958; Woyke, 
oder “ defektiven "Bieneneier gleich wie bei anderen Hymenopteren auf einen ungenügenden Dottergehalt zurückzuführen sei, welcher für eine vollkommene Ausbildung des Embryos nicht ausreiche. Dieses Manko soll infolge einer intraovarialen Resorption von Dotterstoffen entstehen und dann eintreten, wenn die Eier längere Zeit in den Ovarien liegenbleiben, statt abgelegt zu werden. Ganz abgesehen davon, dass Bienenköniginnen, die taube Eier legen, nach erfolgter Begattung mit der Eiablage erfahrungsgemäss ohne merkliche Verzögerung beginnen, müsste die Hypothese von Flanders doch mindestens durch entsprechende mikroskopische Befunde begründet sein, was aber nicht der Fall ist.

Meines Wissens sind solche Eier bislang nur von Nachtsheim (1914) und Hiтchсоск (1956) histologisch untersucht worden. Sie stellten übereinstimmend fest, dass sich die tauben Eier alle zu entwickeln begonnen hatten. Die einen waren in Furchung begriffen, in andern war bereits ein Blastoderm vorhanden oder die Embryogenese mehr oder weniger weitfortgeschritten. Während NACHTSHEIM darauf hinweist, dass in derartigen Eiern sowohl die Furchung als auch die Blastodermbildung regelwidrig verlaufen, sagt Hгтснсоск über seine Befunde nichts Näheres aus. In beiden Publikationen vermisst man leider Bildbeilagen, welche über die abnormen Entwicklungsprozesse einigermassen Aufschluss geben könnten. Als früherer Mitarbeiter der Bienenabteilung der Eidgenössischen Forschungsanstalt für Milchwirtschaft in Liebefeld bei Bern kann ich hier einige Untersuchungsergebnisse mitteilen und mit Originalzeichnungen belegen, welche geeignet sind, unsere bisherigen, recht spärlichen Kenntnisse über das Entwicklungsgeschehen in den tauben Bieneneiern etwas zu erweitern.

In den Jahren 1941-1963 erhielt die Bienenabteilung Liebefeld von Imkern aus 16 verschiedenen Schweizerkantonen nicht weniger als 56 junge, begattete Bienenköniginnen, welche solche Eier legten. Das sind rund $1.4 \%$ von den 3827 Königinnen, die in diesem Zeitraum der Abteilung zur diagnostischen Untersuchung eingesandt wurden. Schon daraus ersieht man, dass die Anomalie keineswegs so selten ist, wie in Bienenzüchterkreisen vielfach angenommen wird. Das geht übrigens auch aus den zahlreichen diesbezüglichen Mitteilungen in der ältern und neueren Bienenliteratur hervor (FYG, 1936; НIтCHCOCK, 1956). Bei 46 $(=82.1 \%)$ der erwähnten 56 Bienenköniginnen fanden sich im Brutnest nur taube Eier. Drei Königinnen $(=5.4 \%)$ legten ausser entwicklungsunfähigen Eiern auch einige, aus welchen normale Arbeiterinnenlarven und Puppen hervorgingen. Im Gelege von 5 weiteren Königinnen $(=8.9 \%)$ entwickelten sich nur die in Drohnenzellen abgelegten Eier und zwar, wie ich mich selber überzeugen konnte, zu normalen männlichen Larven und Puppen. Die restlichen zwei Königinnen $(=3.6 \%$ ) sollen nach den Angaben der Imker zuerst regelrechte Brut erzeugt und erst nach einiger Zeit entwicklungsunfähige Eier gelegt haben.

Die tauben Eier, die ich für die vorliegenden Untersuchungen benützte, stammen von 6 jungen, in den Sommermonaten geschlüpften und richtig begatteten Bienenköniginnen, welche in verschiedenen Jahren gleich nach Empfang jeweils in der üblichen Weise markiert und in kleinere, gesunde Völker des Anstaltsbienenstandes eingeweiselt wurden; hier konnte ihre Eierlage genau kontrolliert werden. Die den Wabenzellen vorsichtig entnommenen Eier wurden sofort in VAN LeEuwen's Pikrinsäuregenisch ${ }^{1}$ oder HoFker-Lösung ${ }^{2}$ fixiert und

1. Zusammensetzung : $1 \%$ Pikrinsäure in abs.Aethylalkohol 12 Teile, $40 \%$ iges Formalin 2 Teile, Chloroform 2 Teile, Eisessig 1 Teil. Fixierungsdauer 6-12 Stunden.

2. $1 \%$ Pikrinsäure in abs.Aethylalkohol 6 Teile, $40 \%$ iges Formalin 1 Teil, Chloroform 1 Teil, Trichloressigsäure 1 Teil. 
über Alkohol von Iangsam steigender Konzentration, Methylbenzoat und Benzol in Paraffin eingebettet. Zur Färbung der lückenlos angefertigten Schnittserien verwendete ich hauptsächlich WeIGERT's Eisenhämatoxylin und Eosin oder Eisenalaun-Hämatoxylin nach HeIOENHAIN. Zu Vergleichszwecken wurden auf dieselbe Weise auch zahlreiche Eier von drei normalen Bienenköniginnen zu mikroskopischen Präparaten verarbeitet. Alle diese mikrotechnischen Prozeduren müssen sehr sorgfältig vorgenommen werden, um die leicht eintretende Schrumpfung der Eier nach Möglichkeit zu vermeiden.

\section{II. - NORMALER VERLAUF DER EMBRYOGENESE BEI DER HONIGBIENE}

Die Embryogenese der Insekten umfasst alle Entwicklungsprozesse von der Eireifung bis zum Ausschlüpfen der Larve aus den Eihüllen. Bei der Honigbiene sind die einzelnen Vorgänge dieses Geschehens von PetrunKewitsch (1901, 1902), Nachtsheim (1912, 1913), Nelson (1915), Schnetter (1935), Müller (1957), Reinhart (1960) und Maul (1967) erforscht und so ausführlich beschrieben worden, dass im Rahmen meiner Mitteilung auf diese Publikationen hingewiesen und auf eine Rekapitulation verzichtet werden kann. Es genügt hier, zu erwähnen, dass die Eireifung nicht in den Ovarien der Bienenkönigin, sondern erst nach der Eiablage in den Wabenzellen stattfindet und die Keimesentwicklung sowohl in den befruchteten als auch unbefruchteten Eiern drei Tage beansprucht. Die Tabelle auf S. 129, welche sich vorwiegend auf die Untersuchungen von NeLson (1915) und SCHNETTER (1935) stützt, gibt Aufschluss über die wichtigsten Phasen und den zeitlichen Ablauf der Embryonalentwicklung bei der Arbeitsbiene. Dieser Überblick erwies sich als sehr nützlich, um bei der Untersuchung der tauben Bieneneier das ungefähre Alter der vorliegenden Entwicklungsstadien zu bestimmen.

\section{III. - DIE KEIMESENTWICKLUNG IN TAUBEN BIENENEIERN : MIKROSKOPISCHE BEFUNDE}

\section{a. - Allgemeines}

Schon bei der ersten Durchsicht der angefertigten Schnittpräparate konnten grosse Unterschiede in der Entwicklung der tauben Bieneneier festgestellt werden. Sie sind einesteils verständlich, wenn man bedenkt, dass die undatierten Eier im Augenblick der Fixierung nicht gleichaltrig waren. Andernteils dürften sie darauf beruhen, dass die Entwicklungskrise in den Eiern der sechs für die vorliegenden Untersuchungen ausgewählten Bienenköniginnen offenbar nicht im gleichen Zeitpunkt der Embryogenese eingetreten ist. Tatsächlich sind die ersten Anzeichen einer Anormogenese in den Eiern der einen Königinnen vielfach schon sehr früh, d. h. vor oder während der 
Blastodermbildung, in den Eiern der andern Königinnen dagegen erst in einer späteren Phase der Keimesentwicklung zu erkennen.

Im folgenden möchte ich zuerst die Struktur der frisch abgelegten tauben Bieneneier und dann einige der beobachteten Anomalien beschreiben, die mir besonders interessant zu sein scheinen. Dabei wird sich öfters Gelegenheit bieten, die abnorme mit der normalen Eientwicklung zu vergleichen.

Arbeitsbiene : Verlauf der Embryogenese

nach NeLson (1915) und SchNETTER (1935)

\begin{tabular}{|c|c|c|}
\hline $\begin{array}{c}\text { Alter } \\
\text { in Stunden } \\
\text { n. Eiablage }\end{array}$ & $\begin{array}{c}\text { Entwicklungs- } \\
\text { phase }\end{array}$ & Kennzeichen \\
\hline $0-31 / 2$ & $\begin{array}{l}\text { Eireifung und } \\
\text { Befruchtung }\end{array}$ & $\begin{array}{l}\text { Reifeteilungen im Richtungsplasma } \\
\text { Kopulation des weiblichen Vorkerns mit einem Spermakern }\end{array}$ \\
\hline $31 / 2-10$ & Furchung & $\begin{array}{l}\text { Furchungskerne zuerst in der Nähe des vordern Eipoles; Bildung einer } \\
\text { anfänglich elliptischen und später keulenförmigen Kernsphäre durch } \\
\text { synchrone Kernvermehrung. Nach 8-10 Stunden erreichen die Furchung- } \\
\text { kerne die Eioberfläche. } \\
\text { Auftreten der primären Dotterzellen (Vitellophagen) }\end{array}$ \\
\hline $12-20$ & $\begin{array}{l}\text { Blastoderm- } \\
\quad \text { bildung }\end{array}$ & $\begin{array}{l}\text { Blastodermbildung durch heterochrone Zellvermehrung; zuerst zweireihige } \\
\text { Anordnung der Blastodermkerne, dann bilden die Blastodermzellen eine } \\
\text { einfache, geschlossene Schicht von prismatischen Zellen. } \\
\text { Degeneration der primären und Auftreten der sekundären Dotterzellen. }\end{array}$ \\
\hline $24-32$ & & $\begin{array}{l}\text { Differenzierung der Zellelemente des Blastoderms. } \\
\text { Vorbereitung für Schichtenbau und Hüllenbildung. }\end{array}$ \\
\hline $34-39$ & Schichtenbau & $\begin{array}{l}\text { Konzentration des Keimmaterials auf der Ventralseite. } \\
\text { Einsenkung der Mittelplatte im vordern Eidrittel und Beginn der Segmen- } \\
\text { tierung. Erscheinen der vordern Mitteldarmanlage und Bildung der vor- } \\
\text { deren Amnionfalte. } \\
\text { Verschluss der Seitenplatten im vordern Eidrittel. } \\
\text { Hintere Mitteldarmanlage entsteht. }\end{array}$ \\
\hline $42-44$ & & $\begin{array}{l}\text { Keimstreif löffelförmig. Vollendeter Verschluss der Seitenplatten. Erschei- } \\
\text { nen der hintern Amnionfalte. } \\
\text { Längenwachstum des schiffchenförmigen Keimstreifes. } \\
\text { Vollendung der Segmentierung. Amnion bildet einen gesehlossenen Sack. }\end{array}$ \\
\hline $44-54$ & $\begin{array}{l}\text { Sonderung der } \\
\text { Organsysteme }\end{array}$ & $\begin{array}{l}\text { Verkürzung des Embryo. Segmentale Organsonderung. } \\
\text { Bildung des Stomo- und Proctodaeums. } \\
\text { Sonderung der mesodermalen Organe. }\end{array}$ \\
\hline $56-72$ & $\begin{array}{l}\text { Histologische } \\
\text { Differen- } \\
\text { zierung }\end{array}$ & $\begin{array}{l}\text { Stadium des fertigen Embryo. } \\
\text { Ventralkrümmung des Embryo. } \\
\text { Nach } 72 \text { Stunden schlüpft der Embryo und wird zur Larve. }\end{array}$ \\
\hline
\end{tabular}




\section{b. - Struktur der frisch abgelegten tauben Eier}

Die Frage, ob sich die normalen und tauben Bieneneier unmittelbar nach der Ablage im feineren Aufbau sichtbar voneinander unterscheiden, stellt sich schon deshalb, weil BETTs $(1923 / 24,1926)$ die Vermutung geäussert hat, dass die aus dem Chorion und der Dotterhaut bestehende Eischale der tauben Eier möglicherweise zu dick sei und so das Atmen und Ausschlüpfen der Embryonen verhindere. LeucKarT hat bereits im Jahre 1871 festgestellt, dass dem nicht so ist. Die Eischale der normalen und tauben Eier ist gleichbeschaffen und bei beiden nur im Bereich der Mikropyle etwas verdickt. Diese Uebereinstimmung beschränkt sich nach meinen Beobachtungen nicht nur auf die Eischale, sondern gilt auch für andere protoplasmatische Bestandteile der Eizelle, so für das corticale, an die dünne Dotterhaut angrenzende Oberflächen - oder Periplasma (“ Keimhautblastem ») und das Reticuloplasma, welches im Nahrungsdotter (Deutoplasma) ein feines Netzwerk bildet. $\mathrm{Ob}$ das gleiche für den Eikern ebenfalls gesagt werden kann, ist unge. wiss, da mein Material zur Abklärung dieser wichtigen Frage nicht ausreicht. Man müsste eine viel grössere Zahl von frisch abgelegten tauben Eiern cytologisch untersuchen, um darüber Aufschluss zu bekommen. Immerhin finden sich in meinen Präparaten einige wenige Eier im Stadium der ersten, im Richtungsplasma vor sich gehenden Reifeteilung; irgendwelche Abweichungen von der Norm sind nicht zu erkennen. Entgegen der Annahme von Flanders (1959) steht zudem fest, dass sich die normalen und tauben Bieneneier in bezug auf den Gehalt an Nahrungsdotter nicht wesentlich voneinander unterscheiden. In beiden enthalten die Hohlräume des Reticuloplasmas sehr wechselnde Mengen von Dotterstoffen. Das ist übrigens auch bei den legereifen Eiern in den Ovarien von normalen, gesunden Bienenköniginnen der Fall und trifft insbesondere für den Glykogengehalt zu.

\section{c. - Furchung}

Im normalerweise polysperm-befruchteten Bienenei beginnt die Furchung unmittelbar nach der Vereinigung des weiblichen und eines männlichen Vorkerns zum ersten, diploiden Furchungskern. Die Kopulation der Pronuclei erfolgt ungefähr zwei Stunden nach der Eiablage in der Nähe des vorderen Eipols und etwas seitlich vom Richtungsplasma. In rascher Folge entsteht dann aus dem ersten Furchungskern durch synchrone Teilungen eine Gruppe von gleichartigen, isopotenten Furchungszellen, die zunächst im vordersten Eidrittel verbleiben und durch Aufnahme von Plasma aus dem Reticulum sichtlich heranwachsen. Mit fortschreitender mitotischer Kernvermehrung verteilen sich die neuentstehenden Furchungszellen im Dotter nach hinten und zeigen vor allem auf der Höhe des grössten Eidurchmessers zugleich die Tendenz, allmählich an die Eiperipherie zu wandern. So entsteht 
vorerst eine elliptische und später birn- oder keulenförmige, fast bis zum hintern Eipol reichende Kernsphäre mit breiterem, rundlichem Vorderund schlankerem Hinterende, welche im Innern in einem stark aufgelockerten deutoplasmatischen Netzwerk als frühzeitig differenzierte Abkömmlinge der Furchungszellen lediglich eine Anzahl primärer Dotterzellen beherbergt. Diesen Vitellophagen, die sich ebenfalls karyokinetisch vermehren, kommt die Aufgabe zu, den Dotter in einen resorbierbaren Zustand überzuführen (MÜLLER, 1957; Reinhardt, 1960). Nach zirka 8 - 10 Stunden erreichen die Furchungszellen die Eioberfläche und zwar wiederum zuerst im Bereich des grössten Eiquerschnittes, wo das präsumptive Differenzierungszentrum (ScHNETTER, 1935) liegt. Damit ist die Furchung abgeschlossen und es beginnt als nächste Phase der Embryonalentwicklung die Blastodermbildung.

Nach meinen Beobachtungen verlaufen die Furchungsprozesse in vielen tauben Bieneneiern ganz normal. Sowohl die Furchungszellen mit Ruhekernen (Abb. 1, A) als auch die Furchungsmitosen (B, 1-6) unterscheiden sich in nichts von jenen in vollwertigen Eiern. Das nämliche gilt vielfach für die Ausbildung der Kernsphäre, die Wanderung der Furchungszellen an die Eiperipherie $\left(\mathrm{C}_{1}, \mathrm{C}_{2}\right)$ und ihre Vereinigung mit dem corticalen Plasma (D). Ebenso häufig kommt es aber vor, dass in den tauben Eiern schon die Bildung der Kernsphäre und das Emporrücken der Furchungskerne nach dem Periplasma eine mehr oder weniger tiefgreifende Störung erleiden. In solchen Fällen sind die Furchungszellen im Eiinnern entweder an verschieden Stellen regellos zusammengeballt (Abb. 2) oder sie verschmelzen in einem gewissen Abstand von der Eioberfläche zu einem bandförmigen, plasmareichen Synzytium (Abb. 3, A und B), welches zahlreiche kleine, pyknotische Kerne enthält und meist eine granulöse Masse (Abb. $3 \mathrm{~B}$, gr) umschliesst. Zuweilen erreicht nur ein Teil der Furchungszellen die Eiperipherie und bildet dann dort nicht selten unregelmässig begrenzte Agglomerate mit oft sichtlich degenerierten Kernen (Abb. 4, $\mathrm{k}$ ). Die sich furchenden tauben Bieneneier zeigen eine solche Vielfalt von derartigen Abweichungen von der Norm, dass hier nur einige wenige als Beispiele ausgewählt werden konnten.

Eine andere, besonders interessante Furchungsanomalie (Abb. 5) konnte ich in mehreren Eiern einer im September 1954 geschlüpften und richtig begatteten Bienenkönigin aus Escholzmatt (Kt. Luzern) feststellen, welche von Anfang an nur taube Eier legte. Während die einen Furchungszellen (1-4) noch annähernd normale Kernteilungen aufweisen, finden sich in vielen andern (5-11) zwei oder mehrere, teils deutlich abnorme Mitosen und isolierte Chromatinpartikel, die im Zellplasma unregelmässig verstreut sind. Einzelne derartige Furchungszellen (11) sind zudem ungewöhnlich gross. Diese regelwidrigen Karyomitosen erinnern in gewissem Grade an multipolare Spindeln, wie sie von NeLson (1915) als äusserst seltene Erscheinung vermutlich in 


\section{ABKÜRZUNGEN IN DEN ABBILDUNGEN 1-12}

Abréviations (Fig. 1-12)

a : vorderer Eipol = Pôle antérieur de l'ouf.

bl : Blastoderm = Blastoderme.

Blz : Blastodermzellen = Cellules blastodermiques.

Bm : Bauchmark = Chaîne nerveuse ventrale.

Cgl : Oberschlundganglion = Ganglion sus-œsophagien.

ch : Chorion.

d : Dotter. Vitellus.

Dfc : Mitteldarmdefekt $=$ Défaut de l'intestin moyen.

dh : Dotterhaut $=$ Membrane vitelline.

$\mathrm{dz} \quad$ : Dotterzellen $=$ Cellules vitellines.

Epd : Epidermis $=$ Epiderme.

f $\quad$ : Furchungszellen $=$ Cellules de segmentation.

gr $\quad$ : granulöse Substanzen $=$ Substances granuleuses.

Ht : hinterer Teil des Keimes = Partie postérieure de l'embryon.

k $\quad$ Kern = Noyau.

$\mathbf{k}_{1} \mathbf{k}_{2}$ : Syncytien mitentarteten Kernen = Syncytium avec noyaux dégénérés.

m : Mitosen $=$ Mitoses.

Md : Mitteldarm $=$ Intestin moyen.

Md.ep : Mitteldarmepithel = Épithélium de l'intestin moyen.

p : hinterer Eipol $=$ Pôle postérieur de l'œuf.

pk : Teilkeim = Embryon partiel.

pl : Plasma.

ppl : Periplasma.

Proc : Hinterdarm (Proctodaeum).

rpl : Reticuloplasma.

Sgl : Unterschlundganglion = Ganglion sous-œsophagien.

Stom : Vorderdarm (Stomodaeum). 

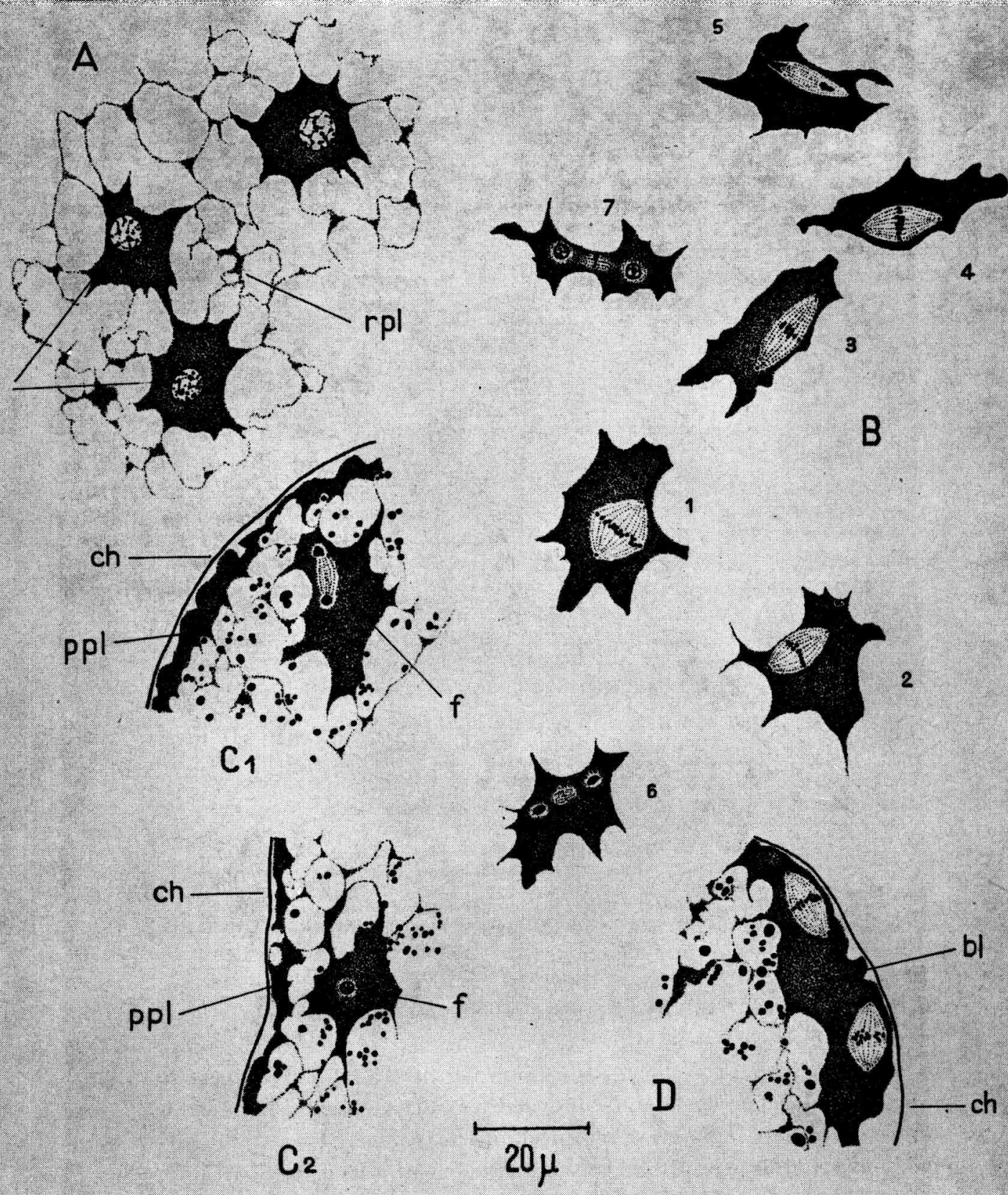

Aв8. 1. - Normale Furchungszellen in tauben Bieneneiern.

A. - Furchungszellen mit Ruhekernen

B. - Furchungsmitosen

$\mathrm{C}_{1} \mathrm{C}_{2}-$ Zur Eioberfläche wandernde Furchungszellen

D. - Vereinigung der Furchungszellen mit dem Periplasma

(Beginn der Blastodermbildung)

Fig. 1. - Cellules de segmentation normales dans des aufs abortifs.

A. - Cellules de segmentation avec noyaux quiescents.

B. - Mitoses de segmentation.

$C_{1} C_{2}$ - Cellules de segmentation migrant vers la surface de l'cuf.

D. - Union des cellules de segmentation avec le périplasma (Début de la formation du blastoderme). 


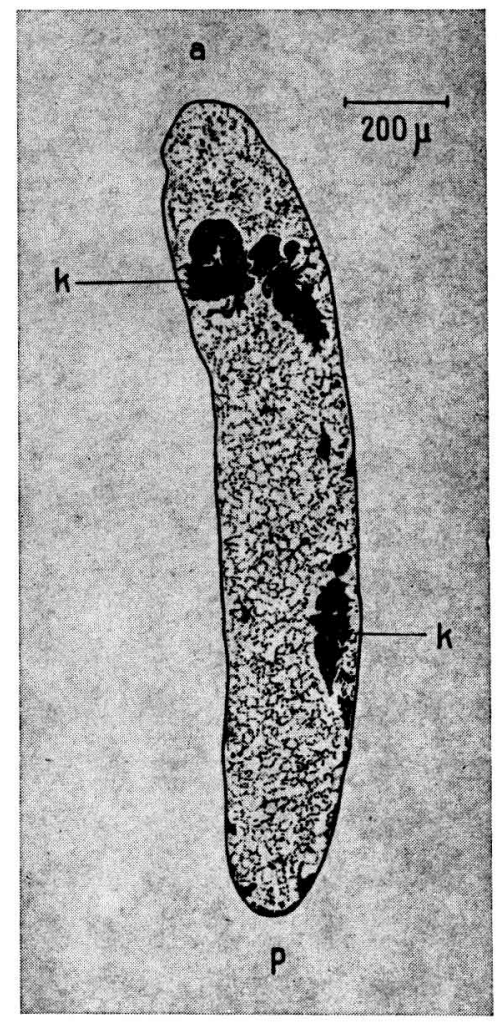

ABB. 2. - Längsschnitt durch ein taubes Bienenei mit regellos agglomerierten Furchungszellen ( $k$ ).

FIG. 2. - Coupe longitudinale à travers un ceuf abortif avec cellules de segmentation (k) agglomérées irrégulièrement.

Dotterzellen und von Reinhardt (1960) in Vitellophagen von künstlich befruchteten Drohneneiern beobachtet wurden. Immerhin besteht insofern ein wesentlicher Unterschied, als es sich bei der in Abb. 5 dargestellten Anomalie um typische Furchungs- und nicht um Dotterzellen handelt. Ob solche abnorme Mitosen in tauben, in Furchung begriffenen Eiern häufiger vorkommen, müsste durch künftige Untersuchungen ermittelt werden.

\section{d. - Blastodermbildung}

Wie die Furchung so kann auch die anschliessende Bildung des Blastoderms in den tauben Bieneneiern entweder in normaler oder regelwidriger Weise erfolgen. Ist das erstere der Fall, so beginnt die Blastodermbildung auf der Höhe des grössten Eiquerschnittes mit dem Eintritt der Furchungskerne in das Periplasma, wobei sie sich sofort mitotisch teilen (Abb. 1, D). 

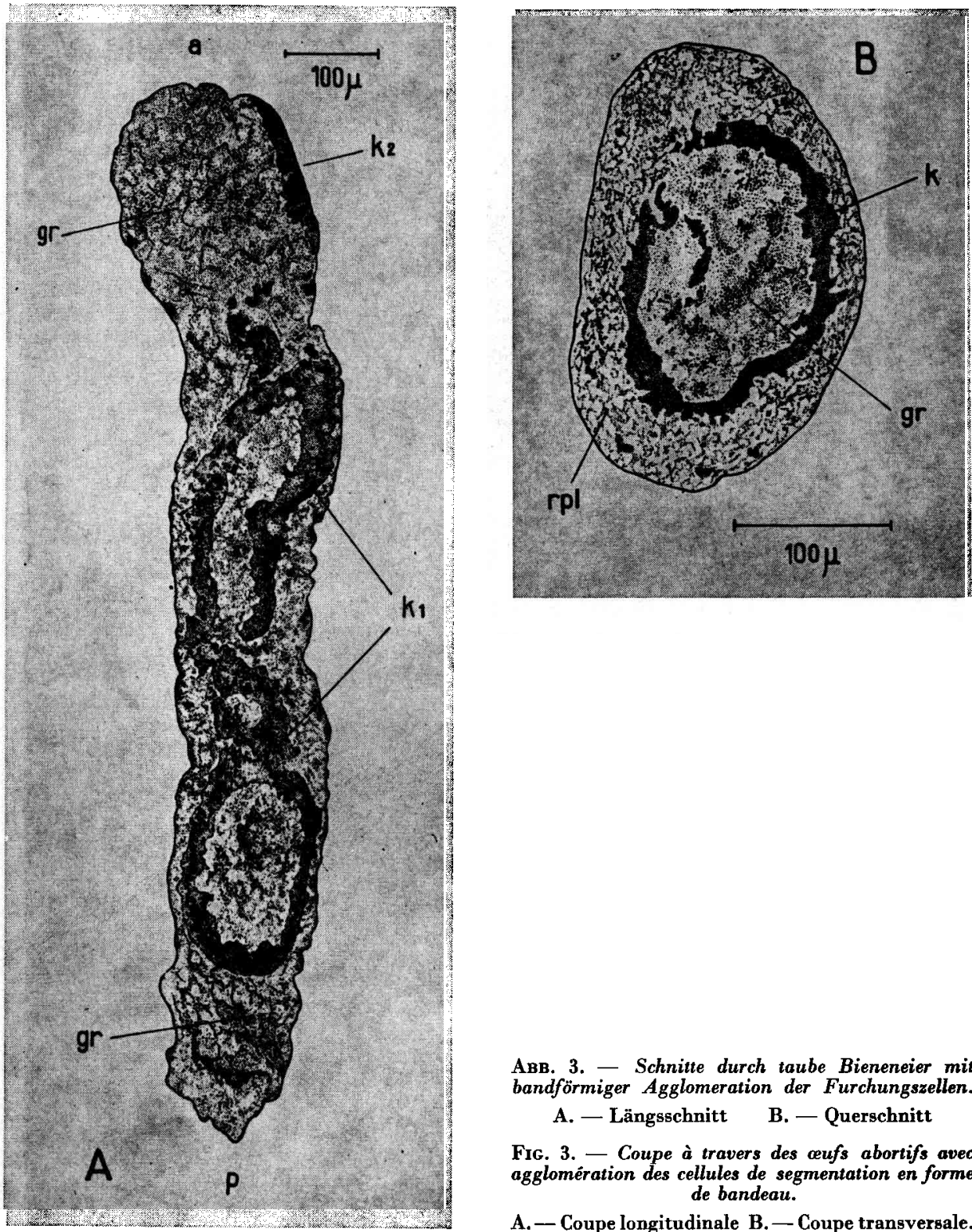

Aв8. 3. - Schnitte durch taube Bieneneier mit bandförmiger Agglomeration der Furchungszellen.
A. - Längsschnitt
B. - Querschnitt

FIG. 3. - Coupe à travers des cufs abortifs avec agglomération des cellules de segmentation en forme de bandeau.

A.-Coupe longitudinale B. - Coupe transversale.

Von da schreitet dieser Prozess nach vorn und hinten über das ganze Ei fort, so dass schliesslich, d.h. etwa 12 - 16 Stunden nach der Eiablage an der Peripherie ein einschichtiges, allseitig geschlossenes Blastoderm entsteht. Dieses Oberflächenepithel hat indessen nicht überall die gleiche Dicke und Beschaffenheit (Abb. 6). Auf der Ventralseite sind die Blastodermzellen wesentlich 
W. FYG

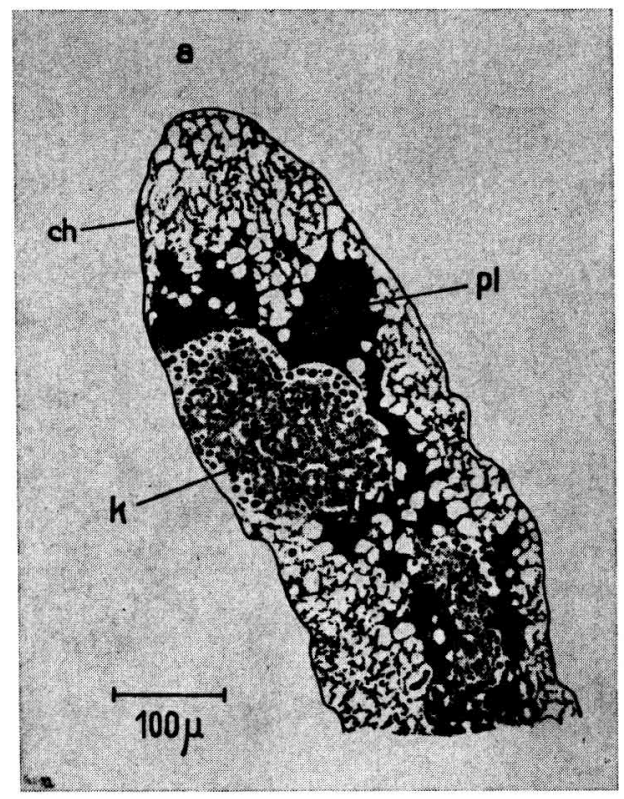

ABB. 4. - Längsschnitt durch die vordere Hälfte eines tauben Bieneneies mit agglomerierten Furchungszellen (k).

Fig. 4. - Coupe longitudinale dans la moitié antérieure d'un cuf abortif avec cellules de segmentation agglomérées $(k)$.

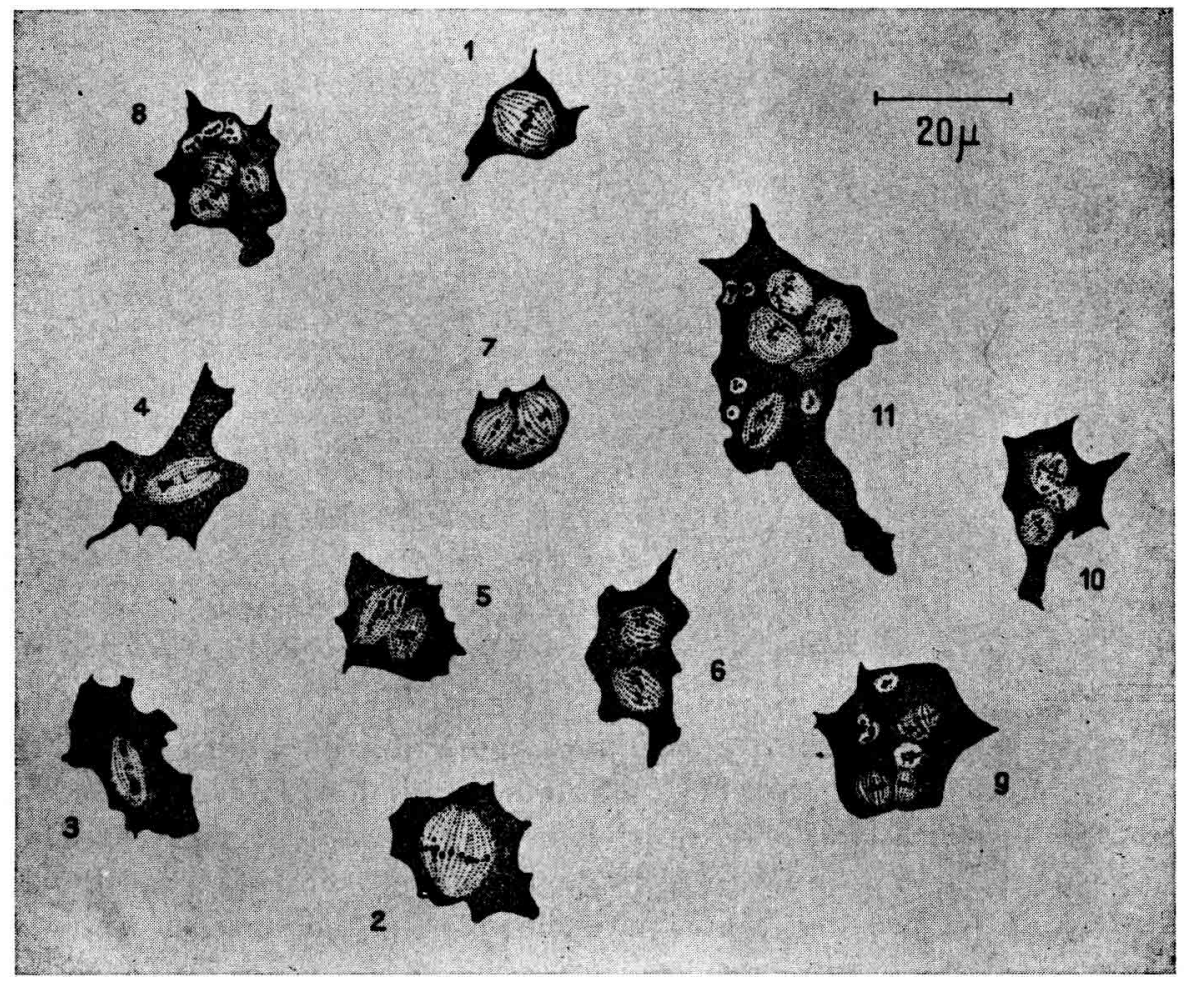

Aв8. 5. - Abnorme Furchungsmitosen in tauben Bieneneiern.

Fig. 5. - Mitoses de segmentation anormales dans des cufs abortifs. 

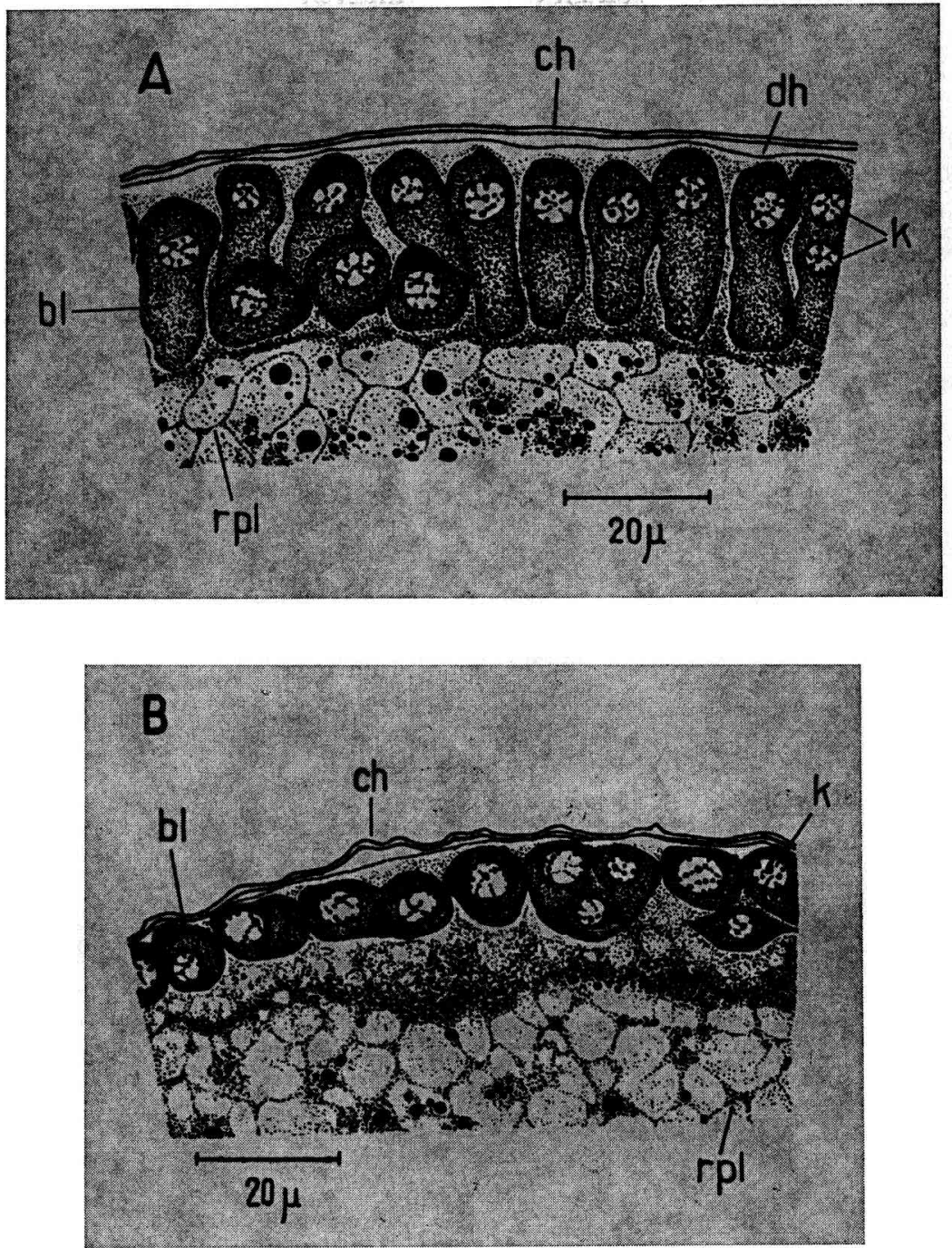

Aвв. 6. - Normale Blastodermbildung in tauben Bieneneiern.
A. - ventrales Blastoderm
B. - dorsales Blastoderm

Fig. 6. - Formation normale du blastoderme dans des oufs abortifs.
A. - blastoderme ventral
B. - blastoderme dorsal.

höher und stehen dichter als auf der Dorsalseite. In der hintern Eiregion finden sich zudem weniger und lockerer angeordnete Zellen als am vorderen Pol. Mit fortsehreitender Entwicklung wird der Unterschied zwischen ventral und dorsal noch ausgeprägter, indem sich die Blastodermzellen auf der 
Ventralseite und lateral davon zu einer verstärkten Schicht zusammenscharen. Hier entsteht in der Folge die embryonale Keimanlage, die allmählich von einer dünnen, aus spindeligen Zellen bestehenden Hülle (Amnion) bedeckt wird.

Soweit ich an Hand meiner Präparate beurteilen kann, sind regelwidrige Blastodermbildungen in tauben Bieneneiern recht häufig und vorwiegend von zweierlei Art. In Abb. 7 ist der eine Typ dargestellt, bei dem ein kern-

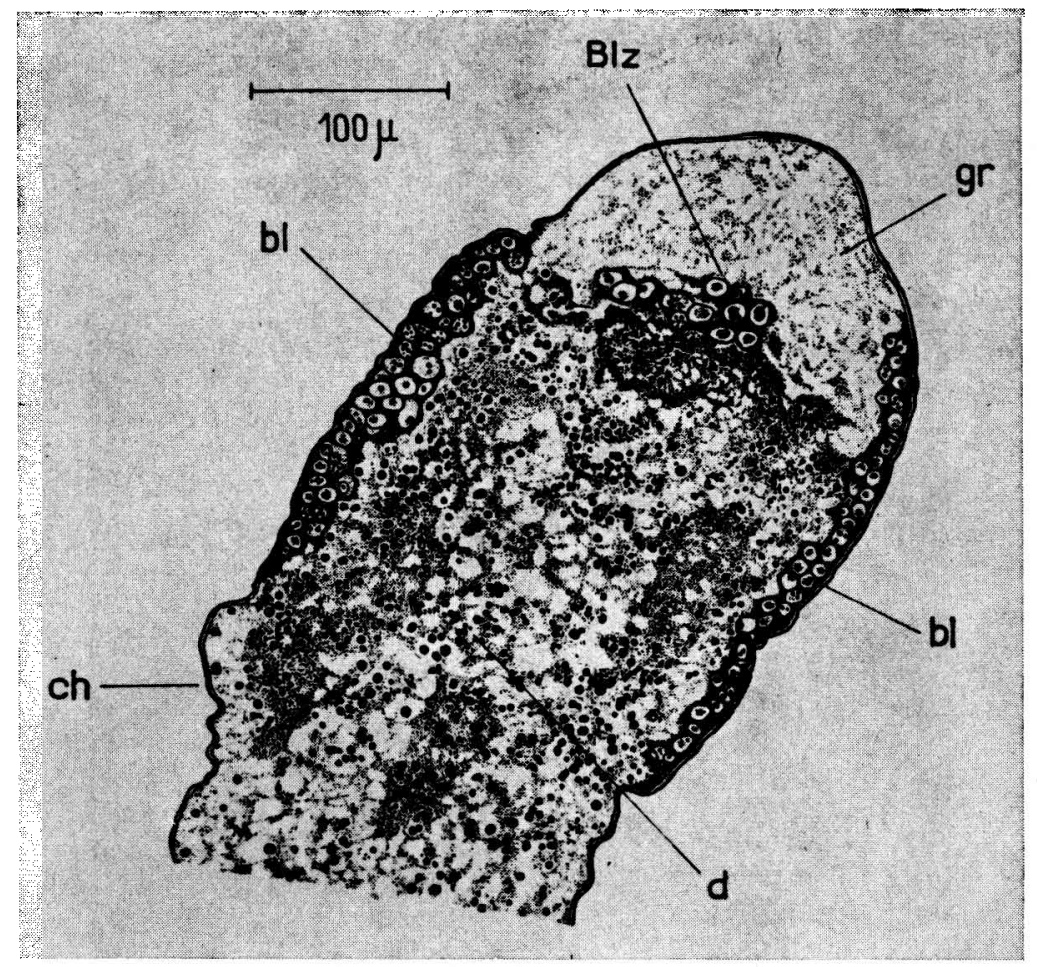

Aвв. 7. - Abnorme Blastodermbildung in taubem Bienenei.

(Längsschnitt)

Fig. 7. - Formation anormale du blastoderme dans des æufs abortifs.

(Coupe longitudinale).

reiches, abnormes Blastoderm (bl) nur in einer ringförmigen Zone im vordersten Eidrittel vorhanden ist und zwar dort, wo die Furchungskerne die Peripherie offenbar zuerst erreicht haben. Ausserdem findet sich ein an den Dotter (d) angrenzendes Agglomerat von Blastoderm- oder Furchungszellen (Blz) etwas hinter dem vordern Eipol, von dem es durch eine Ansammlung von 
feinen Granula (gr) getrennt ist. Noch häufiger sind taube Eier (Abb. 8), in welchen das Blastoderm (bl) lediglich in der vordern, dotterreichen Region eine Kalotte bildet, während die hintern Eiteile sichtlich geschrumpft und frei von Zellen sind. In der Übergangszone enthält das nach hinten auskeilende

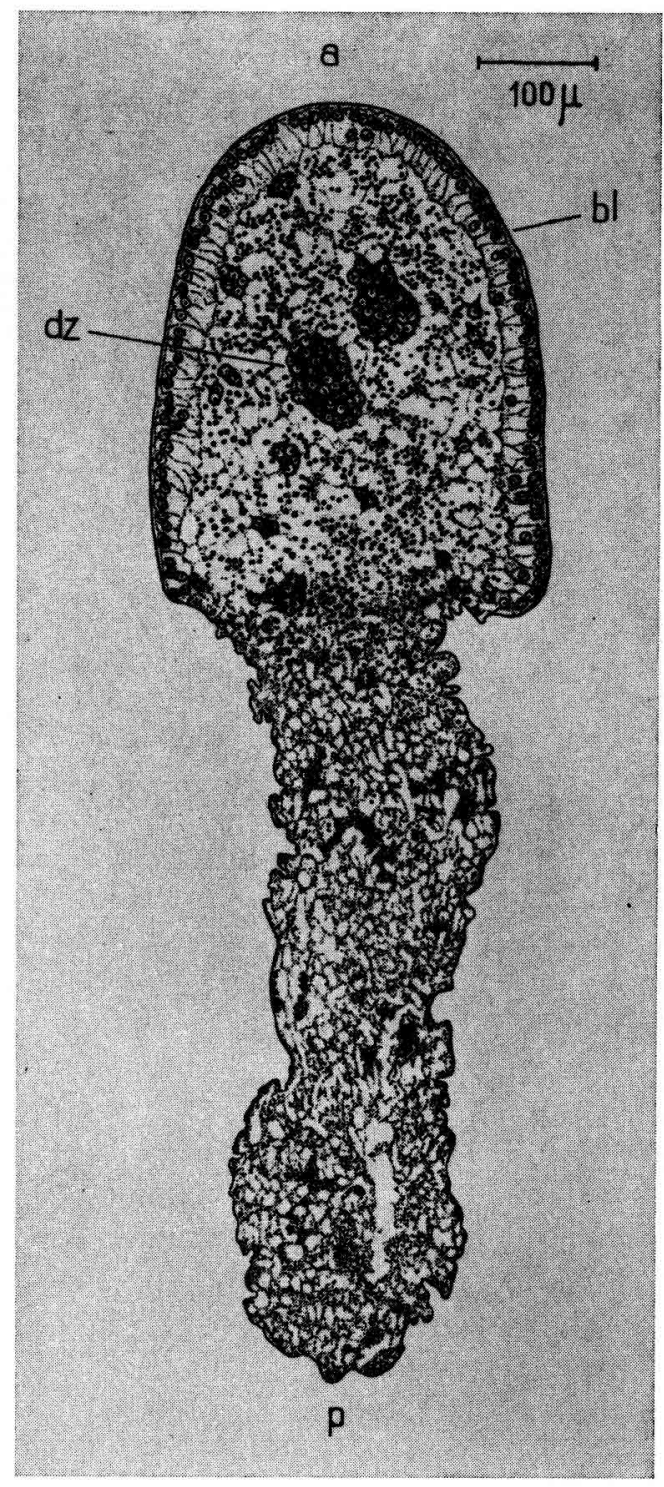

Aвв. 8. - Taubes Bienenei mit Blastodermkalotte. (Längsschnitt)

FIG. 8. - Euf abortif avec calotte blastodermique. (Coupe longitudinale) 
Oberflächenepithel meist deutlich entartete Kerne. Wie in normalen Eiern sind die Dotterzellen (dz) innerhalb der Blastodermkalotte in mehreren, unterschiedlich grossen Gruppen vereinigt und bilden vielkernige Synzytien.

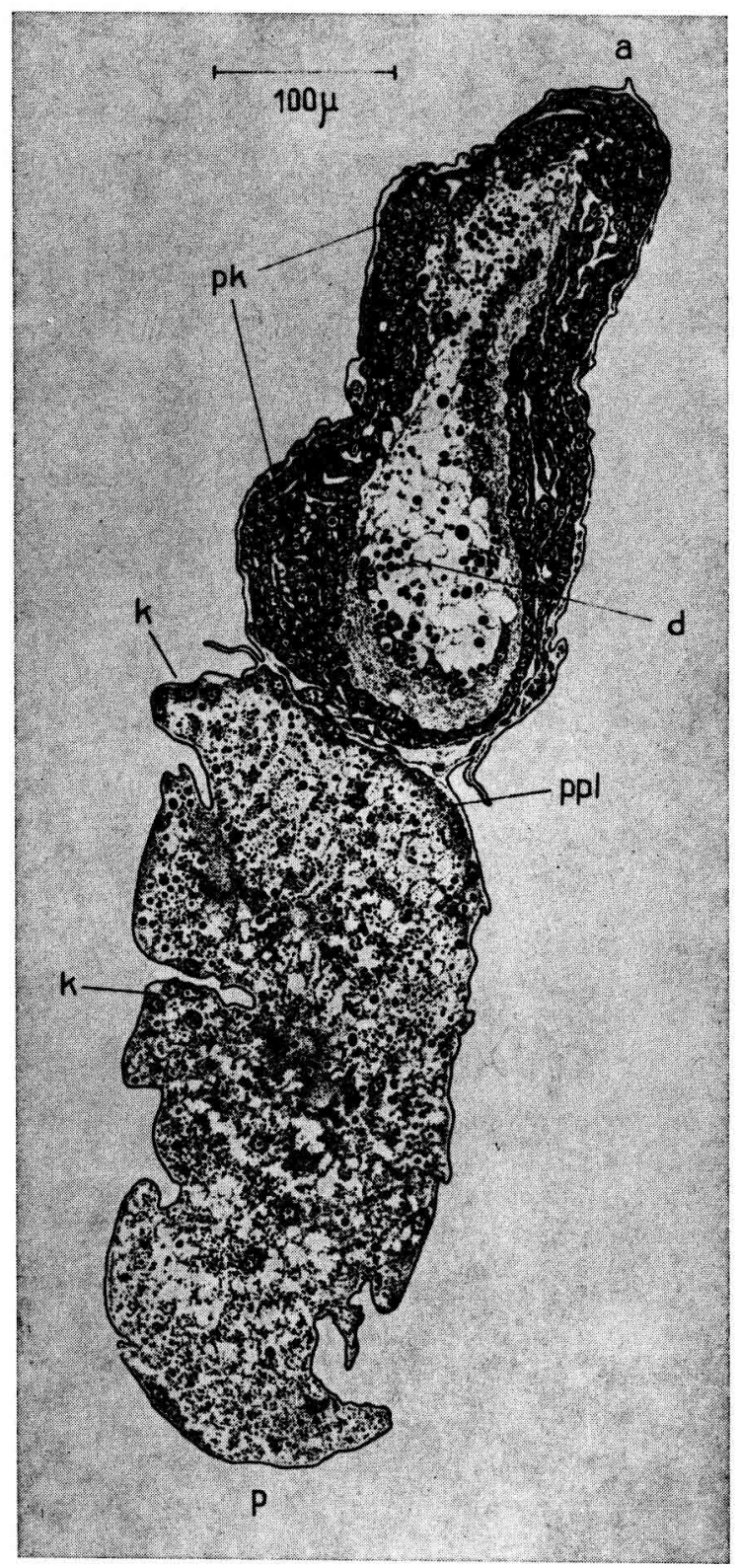

Aвв. 9. - Längsschnitt durch einen Teilkeim.

Fig. 9. - Coupe longitudinale à travers un embryon partiel. 


\section{e. - Teilkeime}

Trotz abnormer Blastodermbildung können sich taube Bieneneier anscheinend noch weiterentwickeln. Dafür sprechen die nachfolgend beschriebenen Teilkeime, welche sich in dem von mir untersuchten Eimaterial in mehreren Exemplaren vorfanden. Einen dieser Fälle veranschaulicht die Abb. 9. Hier ist die Entwicklung in der vorderen Eihälfte schon recht weit fortgeschritten und es scheint, dass in dem in mehrere Schichten gesonderten und zahlreiche Mitosen aufeisenden Keimmaterial (pk) bereits eine Differenzierung der Gewebe begonnen hat. Demgegenüber ist die hintere Eihälfte so gut wie unentwickelt. Im Periplasma (ppl) sind stellenweise nur einige locker angeordnete Furchungskerne (k) vorhanden, ohne dass es zur Bildung eines Blastoderms gekommen wäre. Wie gross der Entwicklungsunterschied in den beiden Eihälften ist, geht auch aus der Abb. 10 hervor, welche von diesem Teilkeim einen stärker vergrösserten Ausschnitt aus der Kontaktzone zeigt.

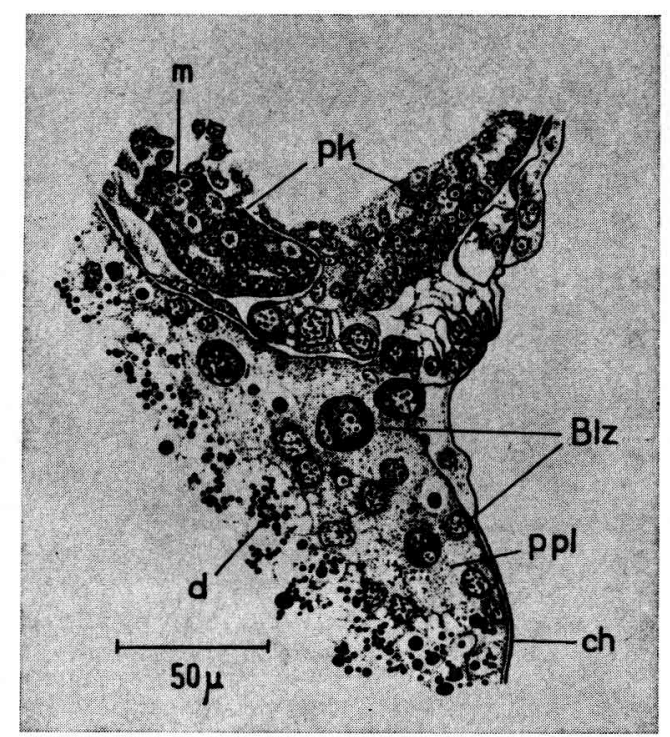

Aвв. 10. - Ausschnitt aus dem in Abb. 9 dargestellten Teilkeim. (stärker vergrössert)

Fic. 10. - Fragment de l'embryon partiel représenté fig. 9 (plus fort grossissement)

Noch ausgeprägter ist die Entwicklungsverschiedenheit in der vordern und hinteren Eiregion bei dem in Abb. 11 dargestellten Teilkeim. Er dürfte ungefähr 50 - 54 Stunden alt sein. Im bedeutend längeren Vorderteil des Keimes (pk) sind schon eine ganze Reihe von Organanlagen leicht zu erkennen, 


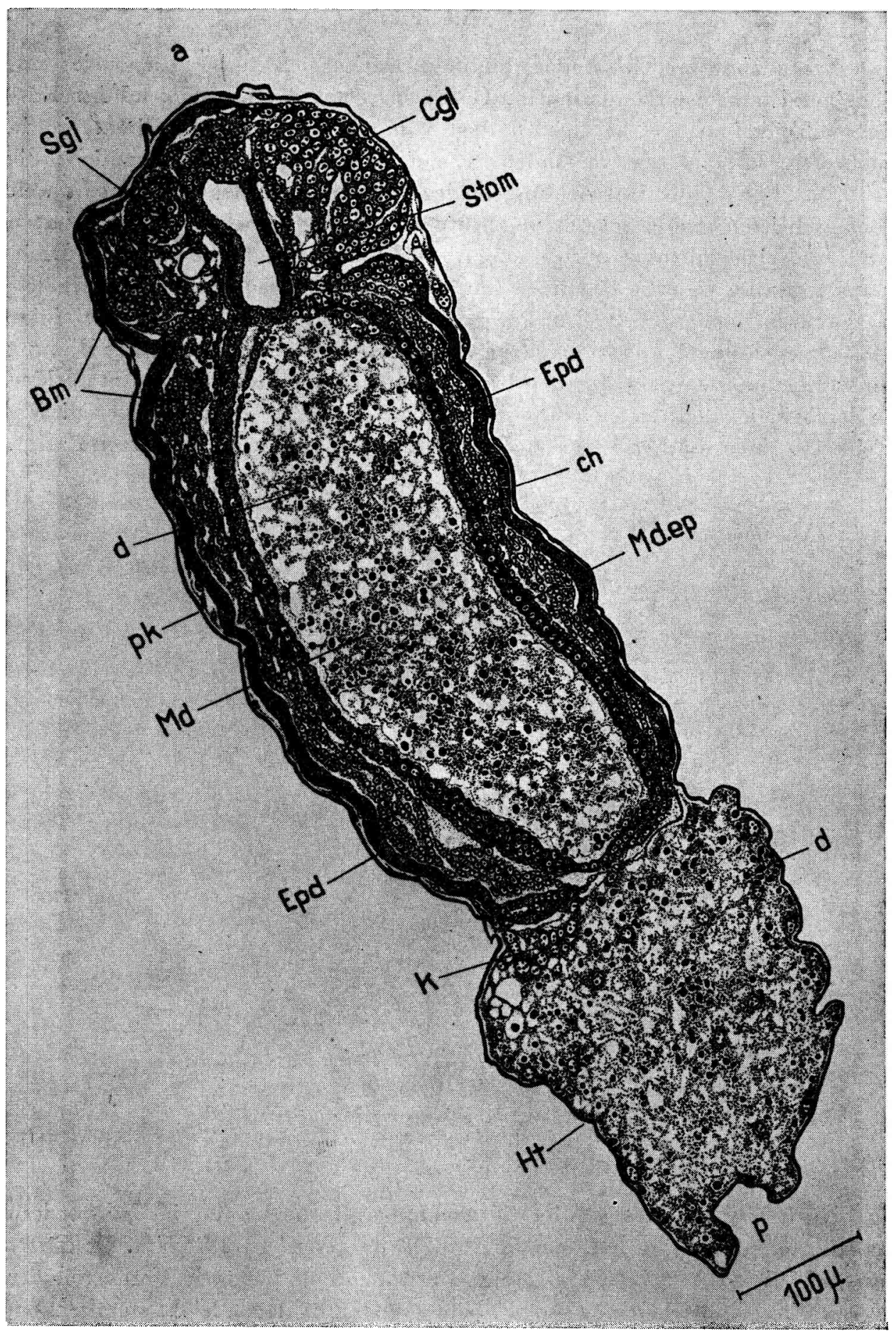

Aв8. 11. - Teilkeim (Längsschnitt).

Fig. 11. - Embryon partiel (Coupe longitudinale). 
so das Ober- und Unterschlundganglion ( $\mathrm{Cgl}, \mathrm{Sgl})$, einzelne Nervenknoten des Bauchmarkes (Bm), dann die Epidermis (Epd) sowie der Vorderdarm (Stom) und der Mitteldarm (Md), welch letzterer noch Dotterreste (d) enthält. Eine Anlage des Proctodaeums fehlt. Im deutlich abgegrenzten Hinterteil (Ht) sind im corticalen Plasma wiederum nur einige Furchungskerne (k) und im Reticuloplasma wenig Dotter vorhanden. Man geht wohl kaum fehl, wenn man annimmt, dass die zwei soeben beschriebenen Teilkeine aus Eiern mit verschieden grossen Blastodermkalotten hervorgegangen sind.

\section{f. - Ganzkeime}

Nach meinen Beobachtungen kann die Embryogenese auch in tauben Bieneneiern öfters so weit fortschreiten, dass fast fertig entwickelte Keime entstehen, welche sich in ihrem Bau mit wenigen Ausnahmen nicht sichtlich von normalen Embryonen unterscheiden. Bei zwei von sechs Bienenköniginnen, welche nur schlüpfunfähige Eier legten, fanden sich im Gelege nicht weniger als $43 \%$, bezw. $57 \%$ derartige Entwicklungsstadien. In den Eiern der vier übrigen Königinnen konnten dagegen nur 4\% oder gar keine Ganzkeime festgestellt werden.

Von den weitgehend fertig entwickelten Embryonen verdient der in Abb. 12 dargestellte Keim deshalb ein besonderes Interesse, weil er einen leicht erkennbaren Defekt (Dfc) des Mitteldarmes (Md) zeigt. Während der embryonale Vorder- und Hinterdarm (Stom, Proc), sowie das Zentralnervensystem ( $\mathrm{Cgl}, \mathrm{Sgl}, \mathrm{Bm})$ und die Körperdecke (Epd) normal ausgebildet sind, ist der Mitteldarm in seinem vorderen Abschnitt auf der Ventralseite offen und nicht wie bei andern, gleichalterigen Embryonen vollständig geschlossen. Infolge dieses Wachstumsdefektes konnte der grösstenteils aus gelösten Dotterresten bestehende Mitteldarminhalt bis in die Nähe des Bauchmarkes (Bm) vordringen. Es ist sehr wohl möglich, dass bei der systematischen Untersuchung einer grösseren Zahl von solchen nicht schlüpfenden Ganzkeimen weitere morphologische Anomalien zu finden wären.

\section{IV. - DISKUSSION}

Aus den vorliegenden histologischen Befunden geht hervor, dass die Keimesentwicklung in den tauben Bieneneiern sehr unterschiedlich verläuft und jedenfalls kein morphologisch einheitliches Schädigungsmuster entsteht. Das trifft zumindest für die Eier der sechs begatteten Bienenköniginnen zu, welche mir das Eimaterial für meine Untersuchungen lieferten. Während die ersten Anzeichen einer Anormogenese bei manchen tauben Bieneneiern schon zur Zeit der Furchung oder Blastodermbildung festzustellen sind, können sich in vielen andern noch verschiedenartige Teilkeime oder schlüpfunfähige Ganzkeime entwickeln, von welch letzteren die meisten keine augenfälligen 


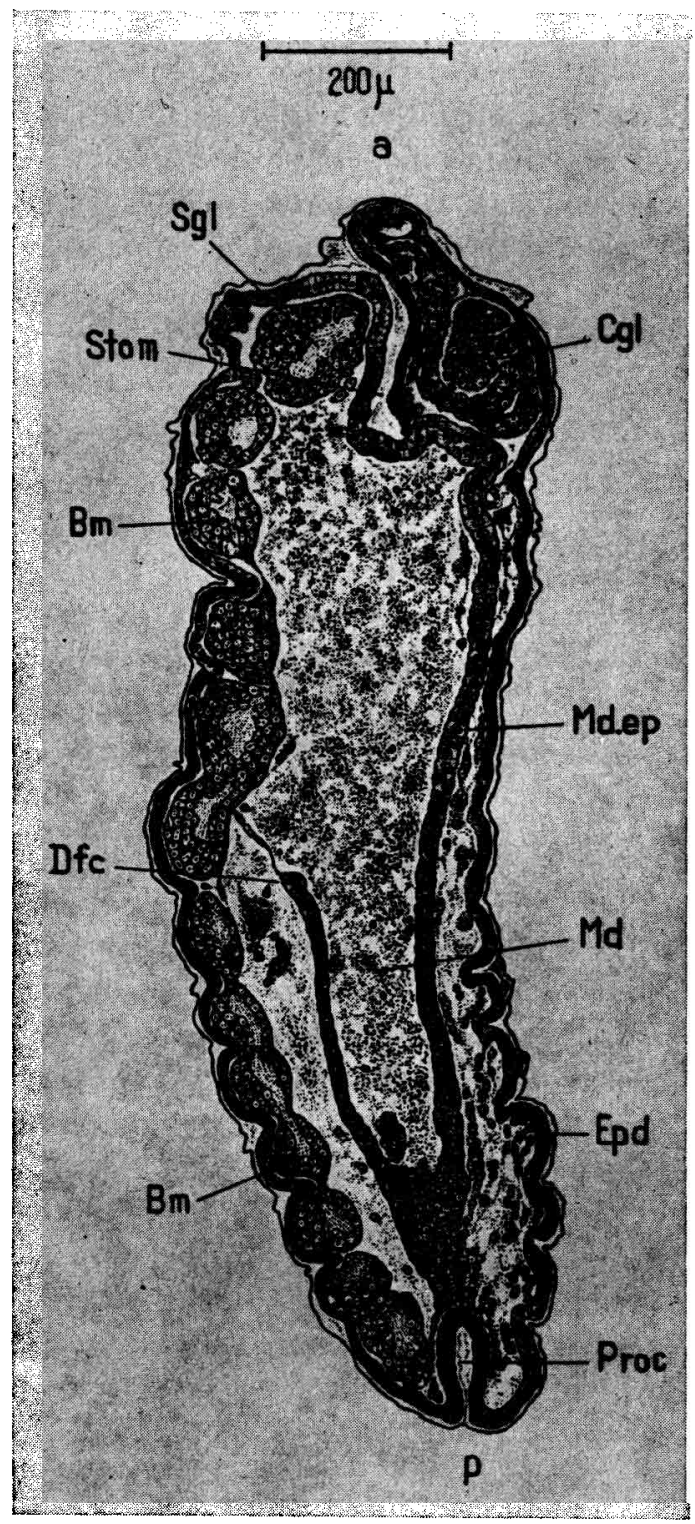

Авв. 12. - Ganzkeim mit Mitteldarmdefelkt. (Längsschnitt)

Fic. 12. - Embryon complet avec défaut de l'intestin moyen. (Coupe longitudinale).

Defekte zeigen. Das schliesst allerdings das Vorhandensein von biochemischen oder funktionellen Störungen bei diesen scheinbar normal ausgebildeten Ganzkeimen nicht aus. Auf alle Fälle steht fest, dass die Fehlentwicklung in 
den tauben Bieneneiern in einer früheren oder späteren Phase der Embryogenese manifest werden kann. $\mathrm{Ob}$ es in der Folge zu einem Entwicklungsstillstand oder sogar zum Absterben der Keime in den Eihüllen kommt, ist ungewiss. Darüber könnte vielleicht die von Reinhardt (1960) angewandte Methode zur Lebendbeobachtung von normalen Bieneneiern Aufschluss geben. Überträgt man solche Eier vorsichtig in $35^{\circ} \mathrm{C}$ warmes Paraffinöl, so entwickeln sie sich bei dieser Temperatur nach den Beobachtungen von ReINHARDT bis zum Schlüpfen der Larven weiter. Würden taube Eier in gleicher Weise behandelt, so müsste es sich dann wohl zeigen, wie weit sich die abnormen Embryonen jeweils zu entwickeln vermögen und ob sie schliesslich nekrotisch werden und so zugrunde gehen.

Der Zweck meiner Mitteilung ist in erster Linie, einige in tauben Bieneneiern festgestellte Anormogenesen bildlich darzustellen und kurz zu beschreiben. Es stellt sich natürlich dennoch die Frage nach der Ursache dieser Fehlentwicklungen und der Schlüpfunfähigkeit der fast reifen Embryonen. Schon in der Einleitung wurde darauf hingewiesen, dass es sich dabei sehr wahrscheinlich um eine erblich bedingte und jedenfalls nicht um eine durch exogene Faktoren verursachte Erscheinung handle. Obwohl es nicht ausgeschlossen ist, dass dabei embryonale Letalfaktoren (HADoRN, 1949, 1955) eine Rolle spielen, erübrigen sich hier in Ermangelung sicherer Anhaltspunkte diesbezügliche und rein hypothetische Erörterungen. Immerhin seien in diesem Zusammenhang zwei Arbeiten erwähnt, die vielleicht wegweisend sind, nämlich diejenige von Maul (1969 und briefliche Mitteilung) und die von Poulson (1940). Maul hat bei der letalen Artkreuzung Apis mellifica L. x Apis cerana Fabr. (Rutrner, 1969) festgestellt, dass sich die unbefruchteten (haploiden) Eier zu normalen männlichen Larven zu entwickeln vermögen. Demgegenüber treten in den befruchteten (diploiden) Eiern bereits im Blastodermstadium schwere Entwicklungsstörungen auf, welche die weitere Embryobildung verhindern und eine gewisse Ähnlichkeit mit den von mir in tauben Bieneneiern beobachteten Anormogenesen zeigen. Das letztere trifft in noch höherem Grade für die Regelwidrigkeiten der Keimesentwicklung zu, die Poulson beim hemizygoten Männchen von Drosophila melanogaster nach Chromosomenstückausfällen im $x$-Chromosom beschrieben hat. Damit soll keineswegs gesagt sein, dass hier wie dort die gleichen Ursachen vorliegen. Maul's und Poulson's Befunde geben aber vielleicht doch die Anregung zu weiteren analogen Untersuchungen bei der Honigbiene.

Als interessante Sonderfälle können jene jungen, begatteten Bienenköniginnen bezeichnet werden, die nicht nur viele taube, sondern in wechsclnder Zahl auch solche Eier legen, aus welchen normale Arbeiterinnenlarven und Puppen hervorgehen. Die Bienenabteilung der Eidgenössischen Forschungsanstalt für Milchwirtschaft in Liebefeld- Bern erhielt in den Jahren 1955- 
1957 gleich drei derartige Königinnen und ihre Gelege zur Untersuchung. In der Imkerliteratur finden sich ebenfalls einige Mitteilungen, die vom nämlichen berichten ${ }^{1}$. Wie kann diese offenbar gar nicht so seltene Anomalie erklärt werden? Wenn die Vermutung richtig ist, dass Letalfaktoren die Fehlentwicklung der tauben Bieneneier bewirken, so könnten die schlüpffähigen weiblichen Larven sogenannte “ Durchbrenner » sein. HAdorN (1955) versteht darunter Tiere, die wohl Träger von Letalfaktoren sind, aber die Entwicklungskrise zu überwinden vermögen und sich deshalb trotz ihrer letalen Veranlagung weiterentwickeln. Nach Hiтchcock (1956) liegt allerdings eine andere Erklärung näher : Bienenköniginnen, welche ausser tauben auch entwicklungs- und schlüpffähige Eier legen, könnten infolge der Mehrfachpaarung in ihrer Samenblase Spermien mit und solche ohne Letalfaktoren beherbergen. Dementsprechend wären die Entwicklungsbedingungen für die befruchteten Eier in erblicher Hinsicht nicht gleich und es würden dann folgerichtig neben abnormen auch normale Keime entstehen. Man muss sich natürlich bewusst sein, dass beide Erklärungen einstweilen nur Hypothesen sind. Und schliesslich ist noch zu bedenken, dass bei den tauben Bieneneiern die Fehlbildungen und die Schlüpfunfähigkeit der Embryonen verschiedene Ursachen haben könnten.

Eingegangen im Februar 1972.

Reçu pour publication en fëvrier 1972.

\section{RÉSUME}

On donne le nom d'œufs abortifs aux oufs d'abeille qui n'éclosent pas ou qui n'éclosent que dans une très faible proportion. Le présent travail décrit brièvement le développement embryonnaire anormal de ces cufs au moyen de dessins originaux. Les recherches histologiques ont montré que les premiers signes d'un développement défectueux peuvent être mis en évidence dans beaucoup d'œufs abortifs dès la segmentation. Ils se manifestent en partie par des mitoses irrégulières (Fig. 5), en partie par une perturbation de la répartition des cellules de segmentation. Dans ce dernier cas il se forme fréquemment à des distances variables de la périphérie de l'œuf des bandes ou des îlots de syncytium plasmatique avec noyaux picnotiques (Fig. 2-4). Souvent, la formation du blastoderme n'a lieu que dans le pôle antérieur de l'œuf (Fig. 7 et 8) et conduit à la formation d'embryons partiels de tailles variables (Fig. 9 et 11). Il n'est pas rare que le développement embryonnaire se poursuive suffisamment pour que se forment des embryons presque achevés mais incapables d'éclore. Dans un cas, on a pu, chez un embryon complet de ce genre (Fig. 11) mettre en évidence un défaut remarquable de l'intestin.

Les présentes constatations histologiques tendent à prouver que la crise de développement dans les œufs d'abeille abortifs se situe visiblement à des moments très différents. La cause de l'anormogénèse et de l'incapacité d'éclosion des embryons en partie développés reste inconnue. On peut penser que l'anomalie est d'origine génétique. Il est, de toute façon, certain qu'il ne s'agit pas d'un phénomène dépendant de facteurs externes.

1. BeTtS, 1923 /24 ; LeUEnBERGER, 1926; IsAaCson, 1932 ; HitchCock, 1956. 


\section{LITERATURVERZEICHINIS}

BETTS A. 1923/24, Eggs that do not hatch. Bee World, 5, 128-129.

Betrs A., 1926, Addled eggs. Bee World, 8, 107.

Claus C. et V. Siebold, C. TH., 1873, Ueber taube Bieneneier. Z. wiss. Zool., 23, 198-210.

FLanders S.E., 1959, Embryonic starvation, an explanation of the defective honey bee egg. J. econ. Entomol., 52, 166-167.

FYG W., 1936, Untersuchungen über die Ursache der Eitaubheit. Schweiz. Bienenztg., 59, 241-252.

Geiger C., 1943, Taube Bieneneier. Bienenmütterchen, $22,37$.

Hachinohe Y. et Jimbu M., 1958, Occurrence of non-viable eggs in the honeybee. Bull. nat. Inst. agric. Sci., Serie G, No. 14, 123-130.

Hadorn E., 1949, Begriffe und Termini zur Systematik der Letalfaktoren. Arch. JuliusKlaus-Stiftung $f$. Vererbungsforsch., 24, 105-113.

HADORN E., 1955, Letalfaktoren in ihrer Bedeutung für Erbpathologie und Genphysiologie der Entwicklung. Georg Thieme Verlag, Stuttgart.

Herlikofer, 1864, Taube Bieneneier. Eichstätter Bienenztg, 20, 169-171.

Hiтcнсоск J.D., 1956, Honey Bee Queens whose eggs all fail to hatch. J.econ. Entomol, 49, 11-44.

IsaACson C.M., 1932, Peculiar behavior of queen : Most of her eggs fail to hatch whille few produce workers. Gleanings in Bee Culture, 60, 685-686.

KleINE G., 1861, Taube Eier. Eichstätter Bienenztg, 17, 174.

LAIDLAW H., Gomes F.P., et KerR W., 1956, Estimation of the number of lethal alleles in a panmictic population of Apis mellifica L. Genetics, 41, 179-188.

LeUckart R., 1871, Kleine Beiträge zur Naturgeschichte der Bienen. Eichstätter Bienenztg., 27, 227-230.

Leuckart R., 1875, U̇ber taube und abortive Bieneneier. Arch. Nat. gesch., 41.

Leuenberger F., 1926, Taube Bieneneier. Schweiz.Bienenztg., 49, 431-434.

MaGkensen O., 1951, Viability and sex-determination in the honey bee (Apis mellifera L.). Genetics, 36, 500-509.

MACKensen O., 1955, Further studies on a lethal series in the honey bee. J. Hered., 46, 72-74.

MaUl V., 1967, Dynamik und Erbverhalten plasmatischer Eibereiche der Honigbiene. Zool. Jb.Anat., 84, 63-166.

Maul V., 1969, Die Ursache der Kreuzungsbarriere zwischen Apis mellifica L. und Apis cerana Fabr. (= Syn. A. indica Fabr.). 2. Eibefruchtung und Embryonalentwicklung. XXII. Intern. Bienenzüchterkongr. München 1969, Apimondia-Verlag Bukarest, 1969, p. 561 .

MüLLER M., 1957, Entwicklung und Bedeutung der Vitellophagen in der Embryonalentwicklung der Honigbiene. Zool. Jb. Abt. Physiol., 67, 111-150.

Nachtsheim H., 1912, Parthenogenese, Eireifung und Geschlechtsbestimmung bei der Honigbiene. Sitzungsber. Ges. Morph. Physiol. München, 28, 22-29.

NaChtsheim M., 1913, Cytologische Studien über die Geschlechtsbestimmung bei der Honigbiene (Apis mellifica L.), Arch. Zellforsch., 11, 169-241.

Nachrsheim M., 1914, Über taube Bieneneier. Münchner Bienenztg., 36, 104-106.

Nelson J.A., 1915, The Embryology of the Honey Bee. Princeton Univ. Press, Princeton.

Petrunkewitsch A., 1901, Die Richtungskörper und ihr Schicksal im befruchteten und unbefruchteten Bienenei. Zool. Jb. Anat. Ont., 14, 573-608. 
Petrunkewitsch A., 1902, Das Schicksal der Richtungskörper im Drohnenei. Ein Beitrag zur Kenntnis der natürlichen Parthenogenese. Zool. Jb. Anat. Ont., 17, 481-516.

Poulson D.F., 1940, The effects of certain x-chromosome deficiencis on the embryonic development of Drosophila melanogaster. J. Exp. Zool., 83, 271-325.

Reinhardt E., 1960, Kernverhältnisse, Eisystem und Entwicklungsweise von Drohnenund Arbeiterinneneiern der Honigbiene (Apis mellifica L.). Zool. Jb. Anat., 78, 167-234.

Rutrnen F., 1968, Génétique. in : Chauvin, R. : Traité de Biologie de l'Abeille. Masson et Cie., Paris, 4, 198-236.

RutrNen F., 1969, Die Ursache der Kreuzungsbarriere zwischen Apis mellifica L. und Apis cerana Fabr. (= Syn. $A$. indica Fabr.) 1. Versuche mit natürlicher und künstlicher Besamung. XXII. Intern. Bienenzüchterkongr. München 1969, Apimondia Verlag Bukarest 1969 , p. 561 .

SchnetTeR M., 1935, Morphologische Untersuchungen über das Differenzierungszentrum in der Embryonalentwicklung der Honigbiene. Z. Morph. Oekol. Tiere, 29, 114-195.

V. Siebold C.Th., 1871, Ủber die Taubheit bei Bieneneiern. Eichstätter Bienenztg., 27, 171-172.

Woyke J., 1962, Hatchability of (c lethal eggs » in two sex-allele fraternity of honeybee. J. apic. Res., 1, 6-13. 